\title{
Microbioma intestinal das aves e sua importância
}

\author{
Intestinal microbiome of birds and its importance \\ Microbioma intestinal de aves y su importancia
}

Recebido: 10/01/2022 | Revisado: 19/01/2022 | Aceito: 21/01/2022 | Publicado: 23/01/2022

Lídia Caroline Ferreira Cruz

ORCID: https://orcid.org/0000-0001-5847-8507

Instituto Federal de Educação, Ciência e Tecnologia Goiano, Brasil

E-mail: lidiaccruz@outlook.com

Thiago Ferreira Costa

ORCID: https://orcid.org/0000-0002-9107-8967

Instituto Federal de Educação, Ciência e Tecnologia Goiano, Brasil

E-mail: thi_costa12@hotmail.com

Stéfane Alves Sampaio

ORCID: https://orcid.org/0000-0002-1302-2001

Instituto Federal de Educação, Ciência e Tecnologia Goiano, Brasil

E-mail: stefanesamp@gmail.com

Nadya Gabrielly Dias da Silva

ORCID: https://orcid.org/0000-0002-6162-9174

Instituto Federal de Educação, Ciência e Tecnologia Goiano, Brasil

E-mail: gabriellynadya@gmail.com

Jessica Martins de Abreu

ORCID: https://orcid.org/0000-0003-1526-7894

Instituto Federal de Educação, Ciência e Tecnologia Goiano, Brasil

E-mail: jessica15_martins@hotmail.com

Kelly Fernanda Borges

ORCID: https://orcid.org/0000-0002-6259-6589

Instituto Federal de Educação, Ciência e Tecnologia Goiano, Brasil

E-mail: kellyfernandaborges2512@gmail.com

Giovanna Macedo Sales

ORCID: https://orcid.org/0000-0003-4026-9023 Instituto Federal de Educação, Ciência e Tecnologia Goiano, Brasil

E-mail: giovannamacedo525@gmail.com

Samantha Leandro de Sousa Andrade Alexandrino

ORCID: https://orcid.org/0000-0001-6849-3081

Instituto Federal de Educação, Ciência e Tecnologia Goiano, Brasil

E-mail: sam87and@gmail.com

Fabiana Ramos dos Santos

ORCID: https://orcid.org/0000-0002-0287-1681

Instituto Federal de Educação, Ciência e Tecnologia Goiano, Brasil

E-mail: fabiana.santos@ifgoiano.edu.br

Cibele Silva Minafra

ORCID: https://orcid.org/0000-0002-4286-2982 Instituto Federal de Educação, Ciência e Tecnologia Goiano, Brasil

E-mail: cibele.minafra@ifgoiano.edu.br

\section{Resumo}

O trato gastrointestinal da ave possui uma diversidade microbiológica, as bactérias de caráter benéfico desempenham papel na digestão e absorção, contribui com o sistema imunológico e na competição contra patógenos que compõe aproximadamente $10 \%$ da microbiota intestinal. Os filos mais abundantes são Actinobacteria, Firmicutes, Fusobacteria e Bacteriodetes, tais microrganismos são essenciais para a saúde da ave. Os microrganismos patogênicos são Proteobacteria, Protozoa, Apicomplexa, Tenericutes e Firmicutes, colonizam o lúmen do trato gastrointestinal da ave sem ocasionar danos, entretanto, quando há disbiose os agentes patogênicos se multiplicam provocando lesões na mucosa intestinal podendo causar distúrbios entéricos, por vezes irreversíveis. A microbiota benéfica é capaz de estimular o sistema imune com a produção de células $\mathrm{T}$, diminuindo o processo inflamatório e a produção de imunoglobulina A, G e M. As Bifidobacterium influenciam a proliferação de macrófagos potencializando o sistema imunológico do animal, além de produzir bacteriocinas que inibe a multiplicação de patógenos (Salmonella spp. e Clostridium perfringens). Os Lactobacillus spp. estimulam a secreção de imunoglobulinas, lactato e acetato e inibem a adesão de patógenos ao epitélio intestinal. Os Bacteroides spp. e Bifidobacterium spp. instituem ácidos graxos voláteis e suprimem a toxicidade da Salmonella spp., Escherichia coli e Campylobacter spp.. A espécie Bacteroides fragilis estimula a produção de células T com o objetivo de diminuir a inflamação. O objetivo principal é 
revisar as informações científicas disponíveis sobre o o microbioma intestinal das aves e sua importância, detalhando sua composição e correlação com o sistema imunológico.

Palavras-chave: Avicultura; Lactobacillus; Microbiota; Sistema Imunológico; Tenericutes.

\begin{abstract}
The bird's gastrointestinal tract has a microbiological diversity, beneficial bacteria play a role in digestion and absorption, contribute to the immune system and competition against pathogens that make up approximately $10 \%$ of the intestinal microbiota. The most abundant phylum are Actinobacteria, Firmicutes, Fusobacteria and Bacteriodetes, such microorganisms are essential for bird health. The pathogenic microorganisms are Proteobacteria, Protozoa, Apicomplexa, Tenericutes and Firmicutes, colonize the lumen of the bird's gastrointestinal tract without causing damage, however, when there is dysbiosis the pathogens multiply causing lesions in the intestinal mucosa and can cause enteric disorders, sometimes irreversible. The beneficial microbiota is able to stimulate the immune system with the production of $\mathrm{T}$ cells, decreasing the inflammatory process and the production of immunoglobulin $\mathrm{A}, \mathrm{G}$ and $\mathrm{M}$. Bifidobacterium influence the proliferation of macrophages, enhancing the animal's immune system, in addition to producing bacteriocins which inhibits the multiplication of pathogens (Salmonella spp. and Clostridium perfringens). Lactobacillus spp. stimulate the secretion of immunoglobulins, lactate and acetate and inhibit the adhesion of pathogens to the intestinal epithelium. Bacteroides spp. and Bifidobacterium spp. they institute volatile fatty acids and suppress the toxicity of Salmonella spp., Escherichia coli and Campylobacter spp. The Bacteroides fragilis species stimulates the production of $\mathrm{T}$ cells in order to reduce inflammation. The main objective is to review the available scientific information about the intestinal microbiome of birds and its importance, detailing its composition and correlation with the immune system.
\end{abstract}

Keywords: Poultry; Lactobacillus; Microbiota; Immune System; Tenericutes.

\title{
Resumen
}

El tracto gastrointestinal de las aves tiene una diversidad microbiológica, las bacterias benéficas juegan un papel en la digestión y absorción, contribuyen al sistema inmunológico y compiten contra los patógenos que constituyen aproximadamente el $10 \%$ de la microbiota intestinal. Los filos más abundantes son Actinobacteria, Firmicutes, Fusobacteria y Bacteriodetes, dichos microorganismos son esenciales para la salud de las aves. Los microorganismos patógenos son Proteobacteria, Protozoa, Apicomplexa, Tenericutes y Firmicutes, colonizan la luz del tracto gastrointestinal de las aves sin causar daño, sin embargo, cuando existe disbiosis los patógenos se multiplican causando lesiones en la mucosa intestinal y pueden ocasionar trastornos entéricos, a veces irreversibles. La microbiota beneficiosa es capaz de estimular el sistema inmunitario con la producción de células $\mathrm{T}$, disminuyendo el proceso inflamatorio y la producción de inmunoglobulina A, G y M. Las Bifidobacterium influyen en la proliferación de macrófagos, potenciando el sistema inmunitario del animal, además de producir bacteriocinas que inhibe la multiplicación de patógenos (Salmonella spp. y Clostridium perfringens). Lactobacillus spp. estimular la secreción de inmunoglobulinas, lactato y acetato e inhibir la adhesión de patógenos al epitelio intestinal. Bacteroides spp. y Bifidobacterium spp. instituyen ácidos grasos volátiles y suprimen la toxicidad de Salmonella spp., Escherichia coli y Campylobacter spp.. La especie Bacteroides fragilis estimula la producción de células T para reducir la inflamación. El objetivo principal es revisar la información científica disponible sobre el microbioma intestinal de las aves y su importancia, detallando su composición y correlación con el sistema inmunológico.

Palabras clave: Avicultura; Lactobacillus; microbiota; Sistema Inmune; Tenericutes.

\section{Introdução}

O segmento intestinal da ave é habitualmente colonizado por microrganismos comensais, simbióticos benéficos e patogênicos, esse microbioma pode chegar a ser duas vezes maior que as células germinais e somáticas do animal. A diversidade microbiológica também influencia na melhora da conversão alimentar (Feitosa, et al., 2020). Lesões nos enterócitos, alterações na altura de vilosidades e na profundidade de criptas podem levar a disbiose dentro do lúmen intestinal favorecendo o microbioma patogênico (Christofoli, et al., 2020). Desde in ovo o TGI de codornas já possui um microbioma, com o seu crescimento o segmento intestinal vai se desenvolvendo e a colonização por microrganismos vai se modificando. As espécies e gêneros que compõem o microbioma intestinal são influenciados por diversos fatores, dentre eles, a nutrição do animal. A microbiota benéfica possui papel essencial no desenvolvimento e saúde da ave, como por exemplo o Enterococcus faecium que possui efeitos probióticos, aumenta a altura dos vilos no íleo e diminui a multiplicação da Salmonella devido a produção de bacteriocina (Kraimi, et al., 2018); (Borda-Molina, et al., 2020). 
O TGI, a microbiota, a camada de muco e o epitélio interagem formando um equilíbrio complexo e dinâmico, garantindo o funcionamento eficiente do sistema digestivo. Quando há uma disbiose do microbioma intestinal, o sistema imunológico é estimulado, portanto, reduzindo o potencial de crescimento do animal. Bactérias diferentes podem desempenhar funções semelhantes, como metabolizar os mesmos substratos e produzir metabólitos semelhantes. Os principais componentes da saúde intestinal da ave são a sua dieta, estrutura e função eficaz da barreira intestinal, microbiota saudável, digestão e absorção, função neuroendócrina e sistema imunológico eficiente. Sendo estes componentes protagonistas da saúde animal e bem-estar (Celi, et al., 2019).

O sistema imunológico da mucosa do TGI é continuamente desafiado pelos antígenos do seu microbima maléfico e, portanto, a presença abundante de microrganismos benéficos e células capazes de reconhecer antígenos bacterianos são indispensáveis. As células dendríticas, linfócitos, macrófagos e mastócitos desempenham um papel fundamental na regulação da comunicação entre a microbiota e o sistema imunológico da mucosa intestinal, com a finalidade de manter um meio microbiológico saudável, para que os patógenos possam ser detectados e neutralizados (Kogut \& Arsenault, 2016). O objetivo principal é revisar as informações científicas disponíveis sobre o o microbioma intestinal das aves e sua importância, detalhando sua composição e correlação com o sistema imunológico.

\section{Metodologia}

$\mathrm{Na}$ elaboração do presente estudo a abordagem metodológica utilizada foi a revisão exploratória, de natureza qualitativa, por meio de pesquisa bibliográfica sobre "Microbioma intestinal e sua importância". Os artigos utilizados para a confecção desta revisão foram retirados de bases indexados: CAPES, PUBMED, SCIELO, SCOPUS, SCIENCE DIRECT, ELSEVIER, sendo recorte temporal das últimas duas décadas. As palavras chaves de pesquisa utilizadas foram: microbiota and chickens/broilers/quails; immune system and broilers/chicken/quails; e pesquisas específicas com os filos e espécies de microrganismos intestinais, priorizando os artigos mais recentes sobre o assunto.

\section{Integridade Intestinal}

A integridade das células que constituem a mucosa intestinal é um dos principais fatores para que a ave tenha uma melhor absorção de nutrientes e consequentemente manter um organismo saudável (Kosmann, 2018). A barreira imune do intestino é o seu próprio epitélio que também é responsável pelo desenvolvimento e crescimento da ave. As células epiteliais do intestino auxiliam na movimentação de íons, fluido, soluto e no transporte de nutrientes do lúmen intestinal para o sistema circulatório (Bogucka, et al., 2017); (Zhou, et al., 2018).

O conjunto de células epiteliais intestinais são sustentadas pela lâmina própria e pela mucosa muscular. As células epiteliais são unidas por junções que conferem uma impermeabilidade a essa camada de células. No local de absorção de nutrientes, a presença de vilosidades e microvilosidades permite ao hospedeiro maximizar a absorção, aumentando a superfície da camada epitelial. A barreira do TGI é composta por uma camada de muco que recobre uma monocamada de células epiteliais e um conjunto subjacente de células compostas por células mesenquimais, células dendríticas, linfócitos e macrófagos que constituem o tecido linfóide associado ao intestino. As células epiteliais intestinais são centrais no sistema imune, pois secretam e regulam a composição da camada de muco, bem como interagem com as células subjacentes. A camada de muco é composta por uma grande variedade de moléculas, sendo a principal delas a mucina sintetizada por células caliciformes (Celi, et al., 2017).

A mucosa intestinal possui vilos que são constituídos por enterócitos, células caliciformes e células enteroendócrinas, que garante a digestão e absorção intestinal. Os enterócitos são responsáveis pela digestão final do alimento e pelo transporte transepitelial dos nutrientes através do lúmen. As células caliciformes secretam glicoproteínas que protegem o epitélio 
intestinal contra os danos enzimáticos durante a digestão. As células enteroendócrinas produzem hormônios peptídicos (gastrina, secretina e colecistoquinina) e monoaminas biogênicas que regulam a digestão, absorção e utilização dos nutrientes (Sommer \& Bäckhed, 2013); (Zebeli, et al., 2018).

O TGI das aves começa seu desenvolvimento desde a fase embrionária e na fase da eclosão ele está totalmente formado, porém, o crescimento celular ainda está em desenvolvimento e sua funcionalidade é comprometida. Entretanto, para que acelere o processo de desenvolvimento intestinal, a oferta de probióticos na dieta das aves nos primeiros dias de vida auxilia na proliferação da microbiota intestinal de forma benéfica (Reis \& Vieites 2019). Em um estudo realizado por Mahrose et al. (2019), a contagem bacteriana total de microrganismos benéficos do intestino delgado e ceco de codornas japonesas aumentaram ao serem suplementadas com probióticos. Dessa maneira também houve uma redução dos microrganismos nocivos ao hospedeiro.

A saúde intestinal é promovida pelo equilíbrio entre a mucosa intestinal, a microbiota e o alimento contido no lúmen, mesmo porque a permeabilidade intestinal é seletiva devido a barreira mecânica formada pela mucosa e pelos enterócitos. $\mathrm{Na}$ produção de aves existem estratégias utilizadas na tentativa de manipulação da microbiota intestinal, a fim de influenciar na saúde, digestão e absorção de nutrientes e melhor desempenho. Uma das principais causas de desequilíbrio do microbioma são as lesões que acometem a mucosa intestinal, deformidade dos vilos intestinais, a deficiência nutricional e o estresse (Guamán, et al., 2017). Os AGCC, como o acetato, propionato e butirato fornecem energia para renovação das células epiteliais a partir da fermentação de carboidratos, algumas bifidobactérias produzem AGCC, contribuindo assim para a integridade intestinal (Binda, et al., 2018).

\section{Biodiversidade da Microbiota}

Os filos mais identificados que colonizam a boca de codornas japonesas são os Firmicutes, Proteobacteria, Bacteroidetes, Actinobacteria, Gemmatimonadetes, Tenericutes e Verrucomicrobia. As bactérias que colonizam o papo, o proventrículo e a moela são as do filo Firmicutes, Bacteroidetes e Proteobactéria. No esôfago, os filos, Firmicutes, o mais abundante, Proteobacteria, Actinobacteria, Tenericutes, Bacteroidetes, Verrucomicrobia e Gemmatimonadetes (Pickler, et al., 2011); (Wilkinson, et al., 2016). No duodeno, jejuno e íleo tem a presença dos microrganismos do filo Proteobacteria, Actinobactéria, Firmicutes, Protozoa e Apicomplexa; no ceco as que pertencem ao filo Actinobacteria, Firmicutes, Proteobactéria e Bacteroidetes (Xiao, et al., 2017). As famílias que colonizam todo o TGI são Bacillaceae, Paenibacillaceae, Staphylococcaceae, Lactobacillaceae, Streptococcaceae, Enterococcaceae, Clostridiaceae, Eubacteriaceae, Microbacteriaceae, Streptomycetaceae, Micrococcaceae, Actinomycetaceae, Bifidobacteriaceae, Enterobacteriaceae, Bacteroidaceae e Flavobacteriaceae (Su, et al., 2014).

O gênero Lactobacillus é predomiante no intestino delgado, já os Megamonas, Faecalibacterium e Bacteroides são dominantes no ceco. A população de Firmicutes, Bacteroidetes e Bacteroides possuem uma população crescente do intestino proximal para distal. Os gêneros Bacteroides, Faecalibacterium, Alistipes, Blautia, Parabacteroides, Anaerostipes e Clostridium são mais abundantes no ceco e no cólon. Contudo, esses microrganismos produzirem AGCC e butirato através das duas principais vias, a via butiril (CoA: acetato $\mathrm{CoA}$ transferase) e a via butirato quinase. Tais substâncias contribuem para a homeostase energética e os processos fisiológicos do hospedeiro (Du, et al., 2020). A superabundância microbiológica a nível de filos e ordem estão ilustradas na figura 1. De modo geral, os gêneros mais abundantes no TGI de codornas japonesas saudáveis são os Lactobacillus, Enterococcus, Megamonas, Bacillus, Faecalibacterium, Alistipes, Bacteroides, Gallicola, Lysinibacillus, Parabacteroides, Corynebacterium, Paenibacillus, Staphylococcus, Blautia, Brevibacostrioides, Clostridium e Anaosfidium (Du, et al., 2020). 
Figura 1. Diversidade da microbiota do TGI de codornas japonesas. a) filo. b) ordem.

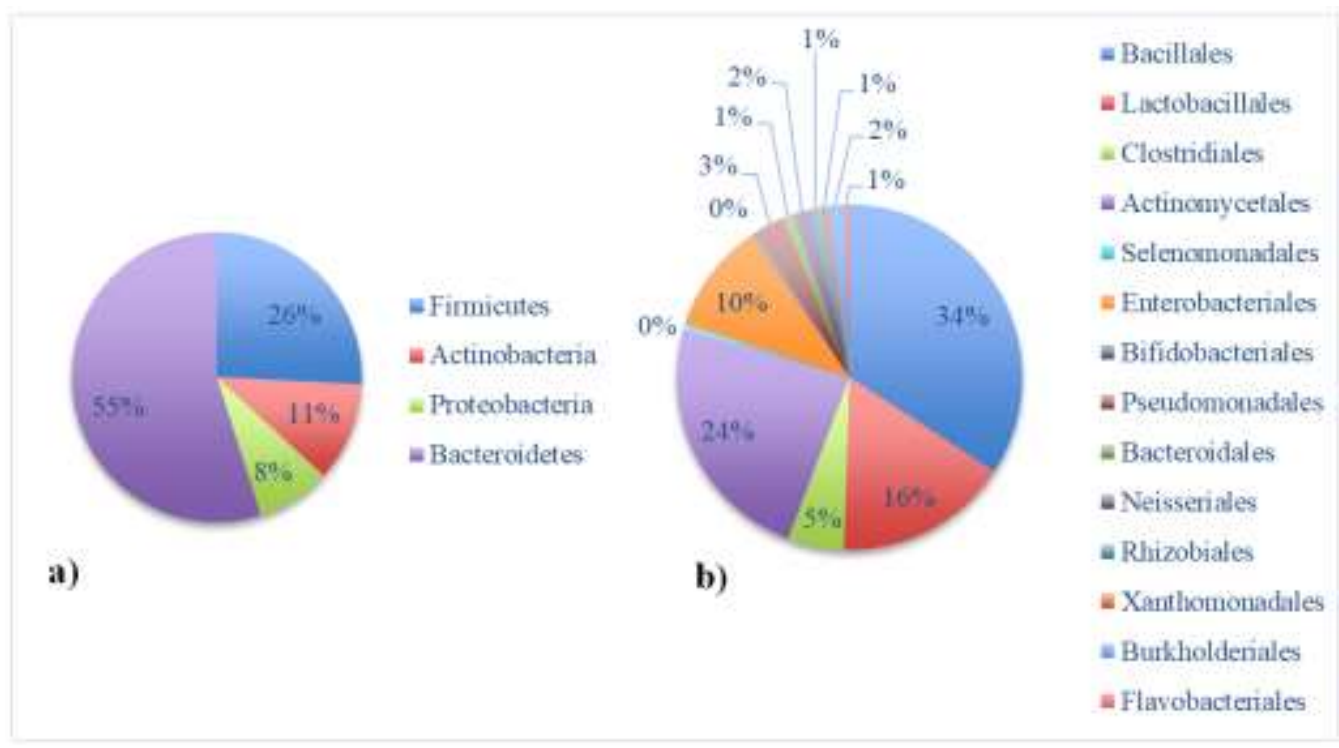

Fonte: Su, et al. (2014).

As codornas do sexo masculino possuem um índice de riqueza em sua microbiota maior que as fêmeas. $\mathrm{O}$ filo Firmicutes possui maior presença nas fêmeas, já a Proteobacteria nos machos. Na boca, os gêneros Lactobacillus são mais presentes nas fêmeas e os Estafilococcus em machos, enquanto que no duodeno o Ruminococcus é em maior quantidade em fêmeas e a família Enterobacteriaceae nos machos. O sexo da ave influencia na composição da microbiota intestinal, como citam o Lactobacillus mais abundante em fêmeas (26\%) do que em machos (22\%), porém, o oposto foi observado com o Estreptococcus com 7,3\% em fêmeas e 9,3\% em machos (Wilkinson, et al., 2016).

\subsection{Microbiota benéfica}

A microbiota benéfica é representada por $90 \%$ dos microrganismos presentes no segmento intestinal e contribuem na absorção e digestão dos nutrientes contidos na dieta e com o sistema imunológico. Portanto, favorece a saúde do organismo da ave, enquanto que a microbiota maléfica predispõe a infecções diminuindo a taxa de crescimento e aumentando a mortalidade. Ademais, os filos que compõem a microbiota benéfica são Actinobacteria, Firmicutes, Fusobacteria e Bacteriodetes (Brian, et al., 2014). As bactérias benéficas do filo Firmicutes produzem ácido lático devido seu metabolismo fermentativo, além de contribuir com a eficiência alimentar possui capacidade de utilizar carboidratos complexos derivados de plantas e produzir butirato. As bactérias do filo Actinobacteria degradam os carboidratos e produzem ácido lático, ácido acético e estão ligadas ao metabolismo dos lipídios e do colesterol (Martínez, et al., 2013); (Apajalahti \& Vienola, 2016).

\subsubsection{Filo Actinobacteria}

As Actinobacterias são gram positivas, filamentosas, possuem alto conteúdo de guanina+citosina $(\mathrm{G}+\mathrm{C})$ em seu genoma, constinuindo um dos maiores filos bacterianos. Seu metabolismo secundário produz substâncias antibióticas, antihelmínticas e antifúngicas. Contém espécies patogênicas (Corynebacterium, Mycobacterium, Nocardia, Propionibacterium e Tropherryma), habitantes do solo (Micromonospora e Streptomyces) e comensais do TGI (Bifidobacterium spp.). São bactérias procarióticas, anaeróbicas, possuem parede celular formada por peptideoglicanos e podem ser heterotróficas. Esse filo possui variedades morfológicas, incluindo cocóide (Micrococcus), bastonetes (Arthrobacter), hifas fragmentadas (Nocardia spp.) e micélios ramificados (Streptomyces) (Barka, et al., 2015); (Salwan \& Sharma, 2020). 
As bactérias do gênero Bifidobacterium colonizam o segmento intestinal das codornas japonesas. Pertencem a classe Actinobacteria, ordem Bifidobacteriales, família Bifidobacteriaceae e reino Bacteria. A multiplicação das Bifidobacterium é influenciada de acordo com a dieta que o animal recebe, os antimicrobianos ingeridos e pelo estresse. São encontradas no intestino delgado e no intestino grosso produzindo ácido acético e ácido lático, induz a produção de imunoglobulina e produzem bacteriocinas (Lugli, et al., 2018).

\subsubsection{Filo Firmicutes}

São gram positivas e pertencem ao reino Bacteria, o gênero que coloniza o TGI é o Lactobacillus, da classe Bacilli, ordem Lactobacillales e família Lactobacillaceae. Possuem metabolismo fermentativo, produzem lactato, etanol, dióxido de carbono, formiato e succinato. Essas substâncias produzidas auxiliam na proliferação das bactérias Veillonella sp., Bacillus sp., Bifidiobacteriuim sp., Bacteriodes sp. Os Lactobacillus sintetizam AGV que impede a toxicidade fúngica reduzindo a concentração de oxigênio (O2) diminuindo o pH e impedindo a difusão de bactérias patogênicas. Entretanto, os ácidos graxos (AG) têm ação benéfica para as bactérias patogênicas do tipo Clostridium perfrigens (Feitosa, et al., 2020).

As espécies do gênero Lactobacillus que abrigam no segmento intestinal das aves são Lactobacillus salivarius, Lactobacillus aviarius, Lactobacillus fermentum, Lactobacillus acidophilus e Lactobacillus reuteri (Okamoto, et al., 2011); (Paixão \& Castro, 2016). Os Lactobacillus sobrevivem sob o pH baixo do proventrículo, inibi o crescimento de Campylobacter em aves, principalmente as espécies $L$. salivarius e L. reuteri. L. acidophilus e L. fermentum são considerados como tendo características probióticas (Goldstein, et al., 2015); (Dez, et al., 2018).

L. Salivarius teve um efeito positivo nas respostas inflamatórias, aumenta a população de bactérias benéficas e reduz bactérias patogênicas na microbiota fecal, aumenta o comprimento das vilosidades e o comprimento da cripta (Sureshkumar, et al., 2020). Além de reduzir o colesterol total e LDL-colesterol, aumenta os trigliceridios, diminui bactérias patogênicas (Escherichia coli), reduz enzimas bacterianas cecais prejudiciais, como $\beta$-glucosidase e $\beta$-glucuronidase (Shokryazdan, et al., 2017).

\subsubsection{Filo Fusobacteria}

São bactérias anaeróbicas gram-negativas, o gênero que pertence a esse filo é o Fusobacterium. Auxiliam na digestão e absorção dos alimentos e no combate a agentes patogênicos que colonizam o ceco. Pertencem ao reino Bacteria, família Bacteroidadeae e ordem Fusobacteriales, assim como as Bacteroides podem se tornar patogênicas em condições específicas. A espécie Fusobacterium mortiferum sintetiza produtos semelhantes a bacteriocina contra a Salmonella enteritidis (Kollarcikova, et al., 2019).

\subsubsection{Filo Bacteroidetes}

As espécies Bacteroides thetaiotaomicron e Bacteroides fragilis estão presentes no ceco das aves, gênero Bacteroides, reino Bacteria, classe Bacteroidetes, ordem Bacteroidales e família Bacteroidaceae. Essas bactérias são anaeróbias gramnegativas comensais. Possuem papel fundamental no desenvolvimento e continuidade da funcionalidade intestinal e no sistema imunológico, porém são oportunistas em casos de lesões na mucosa intestinal e podem apresentar caráter patogênico (Garcia, et al., 2012).

\subsection{Microbioma maléfico}

O microbioma considerado maléfico ao hospedeiro é de aproximadamente $10 \%$ de todo o microbioma intestinal. As afecções do segmento intestinal da ave podem ser acometidas por bactérias, vírus, protozoários e fungos, podendo evoluir de 
uma lesão intestinal para uma septicemia. Devido às alterações o animal apresenta perda do apetite, caquexia, apatia e na maioria das vezes leva a morte (Figueira, et al., 2014).

\subsubsection{Filo Firmicutes}

As principais bactérias pertencem ao gênero Clostridium, são gram-positivas, anaeróbias e produtoras de toxinas. Pertencem ao reino Bacteria, classe Clostridia, ordem Clostridiales e família Clostridiaceae. São encontradas no intestino delgado e ceco, sendo que, a espécie Clostridium colinum causa enterite ulcerativa e a espécie Clostridium perfringens causa enterite necrótica (Barrios, et al., 2013); (Apajalahti \& Vienola, 2016); (Souza, 2017). As espécies do Clostridium produzem toxinas que causam lesões em bactérias benéficas levando a morte celular, consequentemente se proliferam e causa um processo infeccioso. Apresentam ótimo crescimento em temperaturas de $20^{\circ} \mathrm{C}$ a $50^{\circ} \mathrm{C}$ e suportam $\mathrm{pH}$ de 5,0 a 8,0 . Os Clostridium são capazes de fermentar diferentes açúcares, aminoácidos e ácidos nucleicos, seu metabolismo gera ácido acético, ácido butírico e álcool (Albornoz, et al., 2014).

As lesões de enterite necrótica causada pelo Clostridium perfringens acometem na sua maioria o jejuno e íleo, são características histopatológicas da doença as lesões com severa necrose coagulativa aguda na mucosa, geralmente com hemorragia severa (Bortoluzzi, et al., 2019). Podem ser encontrados no solo e em excretas, apesar de serem patogênicos, sua presença e prolongamento no segmento intestinal não é suficiente para prejudicar o animal. Contudo, em casos de deficiência nutricional e diminuição do sistema imunológico da ave, podem promover uma toxicidade entérica e migrar para circulação sistêmica e desencadear doenças (Lacey, et al., 2016).

\subsubsection{Filo Proteobacteria}

As bactérias que habitam o intestino delgado e o ceco das aves são as do gênero Campylobacter, Salmonella e Escherichia, sendo a primeira e a segunda de caráter patogênico. A Campylobacter é microaerófila, ou seja, se multiplicam em concentrações baixas de O2. Tais microrganismos pertencem ao reino Bacteria, classe Epsilonproteobacteria, ordem Campylobacterales, família Campylobacteraceae. Podem causar lesões na mucosa intestinal levando um quadro de enterite hemorrágica. Proteobacteria são microrganismos que provocam a disbiose, seu aumento leva a consequências de drásticas alterações entéricas em aves, prejudicando a saúde do hospedeiro (Sahin, et al., 2015).

A bactéria Campylobacter sobrevive nas fezes das aves depositadas ao solo, contaminando o ambiente, a água e alimentos. É uma bactéria termofílica e quando o animal tem uma carga bacteriana significativa ele apresenta diarreia, perda de peso, inflamação intestinal e hepatite. A transmissão de Campylobacter pode ser vertical quanto horizontal, no meio ambiente por contato oral com as fezes. Essa bactéria pode ultrapassar o epitélio intestinal colonizar o sistema circulatório e outros órgãos, como o baço e fígado (Schets, et al., 2017).

A espécie do gênero Escherichia que acomete as aves é a Eschericia coli, é uma bactéria comensal do TGI que pertence ao reino Monera, classe Gammaproteobacteria, ordem Enterobacteriales e família Enterobacteriaceae. As Enterobacteriaceae são bacilos gram negativos que fermentam glicose são oxidase-negativo, catalase positivo, reduzem nitrato a nitrito e anaeróbicos facultativos. A Escherichia coli é umas das principais bactérias patogênicas e oportunista, protagonista de diversas afecções em animais imunossuprimidos. Está presente no intestino delgado e no ceco das aves em seus primeiros dias de vida (Tanikawa, et al., 2011).

A E. coli possui mais de 80 antígenos $(\mathrm{O}, \mathrm{H} \mathrm{e} \mathrm{K})$, sua membrana externa contém um lipídio A que compõe os lipopolissacarídeos (LPS), sendo importante no seu fator de virulência, seus antígenos e adesinas fimbriais e intimina facilitam sua aderência ao epitélio intestinal e enterócitos. As linhagens de E. coli com caráter patogênico possuem endotoxinas, enterotoxinas, fator citotóxico necrosante (verotoxinas) e hemolisina, tais substâncias auxiliam esse microrganismo em sua 
multiplicação nas mucosas. Os polissacarídeos presentes na cápsula interferem na fagocitose da E. coli, as enterotoxinas afetam a atividade funcional dos enterócitos e as verotoxinas podem produzir lesão celular nos sítios de ação dos mesmos. As verotoxinas absorvidas na corrente sanguínea exercem um efeito deletério nas células endoteliais e inibem a síntese proteica em células eucarióticas (Quinn, et al., 2007); (Zawadzki, 2016).

O gênero Salmonella é aeróbica ou anaeróbica facultativa que pertence ao reino Monera, classe Gammaproteobacteria, ordem Enterobacteriales e família Enterobacteriaceae. A espécie que habita a microbiota intestinal do ceco é a Salmonella enterica que pertence ao reino das Bacterias. A Salmonella entérica possui transmissão horizontal (por eliminação nas fezes) e vertical (ovos e pintainhos) (Oliveira, et al., 2011). Algumas codornas podem se tornar portadoras assintomáticas da Salmonella, seu desempenho produtivo não é prejudicado, porém, continuam contaminando outras aves e produzindo ovos contaminados e pintinhos infectados (Torres, et al., 2016).

\subsubsection{Filo Tenericutes}

Pertencem ao reino Bacteria, são procariontes, não possuem parede celular de peptidioglicano, são comensais do trato gastrointestinal, são parasitas obrigatórios e se assemelham aos vírus devido seu tamanho (300 nm). Podem representar microrganismos de vida livre não associados ao hospedeiro (Skennerton, et al., 2016); (Gupta, et al., 2018). O Mollicutes é a classe mais estudada, que contém gêneros relevantes, incluindo Mycoplasma, Ureaplasma e Acholeplasma. Os Tenericutes tamém podem ser encontrados em uma ampla e diversificada gama de ambientes, como o Izemoplasma em vida livre e o Haloplasma no mar (Wang, et al., 2020).

O gênero Mycoplasma é de importância na avicultura, devido as lesões nas mucosas respiratórias e urogenitais e lesões articulares. As espécies que acometem aves são Mycoplasma gallisepticum, Mycoplasma synoviae e Mycoplasma meleagridis (Machado, et al., 2012); (Emam, et al., 2020). O Mycoplasma synoviae pode causar danos respiratórios subclínicos a agudos e crônicos, sinovite e osteoartrite. Uma das principais causas das alterações patológicas induzidas pelo patógeno é a infiltração de células inflamatórias da camada sinovial (Xu, et al., 2020).

O Mycoplasma gallisepticum pode redurir a atividade da enzima ATPase, levando a uma disfunção do metabolismo energético, uma vez que a enzima ATPase catalisa a decomposição do trifosfato de adenosina (ATP) em adenosina difosfato (ADP) (Ishfaq, et al., 2019). Além de induzir o estresse oxidativo e aumentar o mRNA e o nível de expressão de proteína de genes relacionados à apoptose no baço, burça de fabricius e timo, provocar desequilíbrio da dinâmica mitocondrial de ambos os órgãos e prejudicar sua integridade estrutural. Lesões estas que levam ao dano imunológico, uma vez que, o baço e timo são órgãos linfoides importantes para o sistema imunológico (Zhang, et al., 2020); (Hu, et al., 2021).

\subsubsection{Filo Protozoa}

O parasita presente no intestino delgado da ave é o do gênero Eimeria, reino Protista, classe Rhyzopoda, ordem Coccidia e família Eimeriidae. As espécies que infectam aves são Eimeria acervulina, Eimeria maxima, Eimeria tenella, Eimeria mitis, Eimeria brunetti, Eimeria necatrix e Eimeria praecox (Oden, et al., 2012). É um parasita intracelular obrigatório que induz a resposta imune humoral e mediada, destroem as vilosidades e a mucosa intestinal (Ducatelle, et al., 2018); (Adedokun \& Olojede, 2019). Os danos causados pelo parasita Eimeria possibilita o fornecimento de AA para o crescimento do Clostridium perfringens através dos fluidos teciduais do epitélio danificado. (Golder, et al., 2011); (Shojadoost, et al., 2012). A Eimeria danifica a estrutura intestinal e diminui a população das bactérias pertencentes ao filo Firmicutes. A $E$. tenella provoca lesões nas vilosidades do ceco, desprende as células epiteliais intestinais e aumenta a espessura da porção muscular e da serosa, resultando em extensa perda de células caliciformes e redução da secreção de glicoproteínas. Portanto 
afeta a resposta imunológica da mucosa intestinal. Além de influenciar a colonização de Escherichia e Shigella no ceco (Zhou, et al., 2020).

\subsubsection{Filo Apicomplexa}

Os microrganismos estão presentes no proventrículo, intestino delgado e ceco é o Cryptosporidium, é um parasita coccídio pertencente ao reino Protista, classe Sporozoasida, ordem Eucoccidiorida e família Cryptosporidiidae. A espécie Cryptosporidium meleagridis habita e provoca lesões no intestino delgado, o Cryptosporidium baileyi provoca lesões no ceco e o Cryptosporidium galli infecta o proventrículo. Estes protozoários completam seu ciclo biológico na superfície de células epiteliais do TGI, portanto também são capazes de causar a coccidiose (Helmy, et al., 2017); (Holubova, et al., 2018).

\section{Importância da Microbiota Intestinal no Sistema Imunológico}

As aves não possuem linfonodos, as estruturas que fazem a defesa do organismo são as placas de Peyer, tonsilas cecais e a bursa de Fabricius, no qual captam antígenos disponíveis no segmento intestinal. Os antígenos estimulam as células de defesa em desenvolver imunidade geral inespecífica, células B precursoras de Imunoglobulina A (IgA) e células T. A produção de IgA ocorre devido o estímulo imunológico da mucosa, esses anticorpos bloqueiam os receptores e diminuem o número de bactérias de caráter patogênico na luz do TGI e desencadeiam a ativação de macrófagos (Ajuwon, 2015).

As placas de peyer são compostas por linfócitos T e B localizados na mucosa, estendendo-se até a submucosa no íleo. Os linfócitos da lâmina própria são principalmente linfócitos B secretores de IgA. O papel dessas estruturas imunológicas é endocitar antígenos e transportá-los ao o tecido subjacente, onde são digeridos por macrófagos e células dendríticas. Os anticorpos secretores são transportados para o lúmen através das células epiteliais, e quando expostos a antígenos da placa de peyer, os linfócitos migram para a lâmina própria e o epitélio, onde amadurecem em células citotóxicas. Um grande desafio para o sistema imune intestinal é equilibrar a resposta do hospedeiro aos patógenos e, ao mesmo tempo, não responder aos estímulos derivados de bactérias comensais e também de antígenos alimentares (Celi, et al., 2017).

A resposta imune da mucosa intestinal ao microbioma requer uma capacidade sensorial para diferenciar microrganismos benéficos dos microrganismos patogênicos através do estímulo de secreções de citocinas determinadas por células epiteliais. A microbiota benéfica auxilia na regulação do sistema imunológico, estimula a produção de mucina e a proliferação de células epiteliais (Kosmann, 2018). As bactérias patogênicas para começar seu processo infeccioso precisam se aderir à superfície epitelial, essa adesão ocorre através das glicoproteínas (lectinas) (Hazrati, et al., 2020). As Bifidobacterium influenciam a proliferação de macrófagos potencializando o sistema imunológico do animal, além de produzir bacteriocinas que inibe a multiplicação de patógenos (Salmonella spp. e Clostridium perfringens). Contudo, estimulam a produção de vitamina do complexo B, contribui na reparação da microbiota após ingestão prolongada de antibióticos e auxilia na digestão e absorção de nutrientes. Sua colonização no TGI reflete em um organismo saudável. As altas concentrações de acetato produzido pelas bifidobactérias protegem o hospedeiro contra infecções entéricas (Binda, et al., 2018).

Os ácidos secretados pelas Bifidobacterium e Lactobacillos diminuem o $\mathrm{pH}$ do intestino grosso e inibe o crescimento de agentes patogênicos. Os Lactobacillus spp. estimulam a secreção de imunoglobulinas, lactato e acetato. Os Bacteroides spp. e Bifidobacterium spp. instituem AGV e suprimem a toxicidade da Salmonella spp., Escherichia coli e Campylobacter spp. (Brian, et al., 2014). A espécie Bacteroides fragilis possui polissacarídeo capsular imunomodulador A que estimulam a produção de células T com o objetivo de diminuir a inflamação (Kogut, 2019). As células T são capazes de regular e secretar a interleucina 10 (IL-10), que restringe a inflamação patogênica do intestino, principalmente contra infecções virais (Blandford, et al., 2019); (Ramakrishna, et al., 2019). A espécie Fusobacterium mortiferum sintetiza produtos semelhantes a bacteriocina contra a Salmonella enteritidis (Kollarcikova, et al., 2019). 
Os ácidos biliares excretados pelo fígado são metabolizados pela microbiota intestinal formando ácidos biliares secundário ao que regulam vias imunológicas e metabólicas do animal. Esses ácidos biliares secundários podem inibir a multiplicação dos Clostridium difficile (Brandl, et al., 2017). Ainda, as células imunológicas do TGI se comunicam com a medula óssea e o baço, fazendo com que haja uma maturação do sistema imunológico do hospedeiro, estimulando respostas específicas ao antígeno que são absorvidas pelas células dendríticas residentes (Adedokun \& Olojede, 2019).

O jejum prolongado possui influência sob a microbiota benéfica do ceco, diminuindo a abundância de Lactobacillus, sendo essas bactérias auxiliares na sintetização de AGCC, fonte de energia para a ave. Os Lactobacillus transformam carboidratos em ácido lático, inibem a adesão do agente patogênico ao epitélio intestinal e diminui o pH do íleo (Borda-Molina, et al., 2020).

\section{Considerações Finais}

$\mathrm{O}$ trato gastrointestinal das aves possui uma diversidade de microrganismos que contribuem ou afetam negativamente sua saúde. A microbiota benéfica auxilia em toda fisiologia da digestão dos alimentos e induzem grande parte da resposta imunológica do organismo. Na eventualidade de uma disbiose, os microrganismos maléficos multiplicam provocando lesões no epitélio intestinal rompendo todo equilíbrio da microbiota com o sistema imunológico. Manter a integridade do epitélio intestinal é um dos principais fatores contribuintes para saúde da ave, sendo a mesma promovida pela mucosa intestinal, alimento ingerido e microrganismos entéricos.

Devido à complexidade da relação microbiota e saúde intestinal das aves, são necessários mais estudos sobre a relação microbiota-nutrição-saúde e composição da comunidade microbiana frente a desafios sanitários, bem como os efeitos nutricionais sobre a composição do microbioma da luz intestinal.

\section{Referências}

Adedokun, S. A. \& Olojede, O. C. (2019). Optimizing gastrointestinal integrity in poultry: the role of nutrients and feed additives. Frontiers in Veterinary Science, 5, 348. https://doi.org/10.3389 / fvets.2018.00348

Ajuwon, K. M. (2015). Toward a better understanding of mechanisms of probiotics and prebiotics action in poultry species. Journal Applied Poultry Research, 25, 277-283. https://doi.org/10.3382/japr/pfv074

Albornoz, L. A. L., Nakano, V., \& Campos, M. J. A. (2014). Clostridium perfringens e a enterite necrótica em frangos: principais fatores de virulência, genéticos e moleculares. Brazilian Journal of Veterinary Research and Animal Science, 51(3), 178-193. http://dx.doi.org/10.11606/issn.1678-4456.v51i3p178193

Apajalahti, J., \& Vienola, K. (2016). Interação entre microbiota intestinal de frango e digestão de proteínas. Animal Feed Science and Technology, 221. https://doi.org/323- 330. 10.1016/j.anifeedsci.2016.05.004

Barka, E. A., Vatsa, P., Sanchez, L., Gaveau-Vaillant, N., Jacquard, C., Meier-Kolthoff, J. P., Klenk, H. P., Clément, C., Ouhdouch, Y., \& Wezel, G. P. (2015). Taxonomy, Physiology, and Natural Products of Actinobacteria. Microbiology and Molecular Biology Reviews, 80(1), 1-43. https://doi.org/10.1128/MMBR.00019-15

Barrios, M. A., Saini, K. J., Rude, C. M., Beyer, R. S., Fung, D. Y. C., Bavaresco, C., Nunes, A. P., Forgiarini, J., Alves, D. A., Xavier, E. G., Lopes, D. C. N., \& Rol, V. F. B. (2019). Morfometria intestinal e qualidade óssea de codornas Japonesas alimentadas por um período prolongado com produtos do óleo de soja. Archives of Veterinary Science, 24, 72-82.

Binda, C., Lopetuso, L. R., Rizzatti, G., Gibiino, G., Cennamo, V., \& Gasbarrini, A. (2018). Actinobacteria: A relevant minority for the maintenance of gut homeostasis. Digestive and Liver Disease, 50(5), 421-428. https://doi.org/10.1016/j.dld.2018.02.012

Blandford, L. E., Johnston, E. L., Sanderson, J. D., Wade, W. G., \& Lax, A. J. (2019). Promoter orientation of the immunomodulatory Bacteroides fragilis capsular polysaccharide A (PSA) is off in individuals with inflammatory bowel disease (IBD). Gut Microbes, 10(5), 569-577. https://doi.org/10.1080/19490976.2018.1560755

Bogucka, J., Dankowiakowska, A., Elminowska-Wenda, G., Sobolewska, A., Jankowski, J., Szpinda, M., \& Bednarczyk, M. (2017). Performance and small intestine morphology and ultrastructure of male broilers injected in ovo with bioactive substances, Annals of Animal Science, 17, 179-195. https://doi.org/10.1515/aoas-2016-0048

Borda-Molina, D., Roth, C., Hérnandez-Arriaga, A., Rissi, D., Vollmar, S., Rodehutscord, M., Bennewitz, J., \& Camarinha-Silva, A. (2020). Effects on the Ileal Microbiota of Phosphorus and Calcium Utilization, Bird Performance, and Gender in Japanese Quail. Animals, 10, 885. https://doi.org/10.3390/ani10050885 
Bortoluzzi, C., Vieira, B. S., Hofacre, C. \& Applegate, T. J. (2019). Effect of different challenge models to induce necrotic enteritis on the growth performance and intestinal microbiota of broiler chickens. Poultry Science, 98, 2800-2812. https://doi.org/10.3382 / ps / pez084

Brandl, K., Kumar, V. \& Eckmann, L. (2017). Gut-liver axis at the frontier of host-microbial interactions. American Journal of Physiology-Gastrointestinal and Liver Physiology, 312, 413-419. https://doi.org/10.1152 / ajpgi.00361.2016

Brian, B., Oakley, H. S., Lillehoj, M. H., Kogut, W. K., Kim, J. J., Maurer, A., Pedroso, M. D., Lee, S. R., Collet, T. J., \& Johnson, N. A. C. (2014). O microbioma gastrointestinal de frango. FEMS: Microbiology Letters, 360, 100-112.

Celi, P., Cowieson, A. J., Fru-Nji, F., Steinert, R. E., Kluenter, A. M., \& Verlhac, V. (2017). Gastrointestinal functionality in animal nutrition and health: New opportunities for sustainable animal production. Animal Feed Science and Technology, 234, 88-100. https://doi.org/10.1016/j.anifeedsci.2017.09.012

Celi, P., Verlhac, V., Calvo, E. P., Schmeisser, J., \& Kluenter, A. M. (2019). Biomarkers of gastrointestinal functionality in animal nutrition and health. Animal Feed Science and Technology, 250, 9-31. https://doi.org/10.1016/j.anifeedsci.2018.07.012

Christofoli, M., Souza, C. S., Costa, T. F., Alexandrino, S. L. S. A., Faria, P. P., Rezende, C. S. M., Santos, F. R., Minafra, C. S., \& Pereira, P. S. (2020). Microbiota intestinal benéfica e prejudicial na avicultura: Revisão. Research, Society and Development, 9, e43973667. http://dx.doi.org/10.33448/rsdv9i7.3667

Dez, M., Nowaczek, A., Urban-Chmiel, R., Stępień-Pyśniak, D., \& Wernicki, A. (2018). Probiotic potential of Lactobacillus isolates of chicken origin with anti-Campylobacter activity. Journal Veterinary Medical Science, 80(8), 1195-1203. https://doi.org/10.1080 / 10.1292/jvms.18-0092

Du, X., Xiang, Y., Lou, F., Tu, P., Zhang, X., Hu, X., Lyu, W., \& Xiao, Y. (2020). Microbial Community and Short-Chain Fatty Acid Mapping in the Intestinal Tract of Quail. Animals (Basel), 10(6), 1006. https://doi.org/10.3390/ani10061006

Ducatelle, R., Goossens, E., Meyer, F., Eeckhaut, V., Antonissen, G., Haesebrouck, F., \& Immerseel, F. V. (2018). Biomarkers for monitoring intestinal health in poultry: present status and future perspectives. Veterinary Research, 49, 43. https://doi.org/10.1186 / s13567-018-0538-6

Emam, M., Hashem, Y. M., El-Hariri, M., \& Jakeen, El-Jakee. (2020). Detection and antibiotic resistance of Mycoplasma gallisepticum and Mycoplasma synoviae among chicken flocks in Egypt. Veterinary World, 13(7), 1410-1416. http://dx.doi.org/10.14202/vetworld.2020.1410-1416

Feitosa, T. J. O., Silva, C. E., Souza, R. G., Lima, C. D. S., Gurgel, A. C., Oliveira, L. L. G., Nóbrega, J. G. S., Carvalho Jr, J. E. M., Melo, F. O., Santos, W. B. M., Feitoza, T. O., Costa, T. F., Brandão, P. A., \& Minafra, C. S. (2020). Microbiota intestinal das aves de produção: revisão bibliográfica. Research, Society and Development, 9, e42952779. http://dx.doi.org/10.33448/rsd-v9i5.2779

Figueira, S. V., Mota, B. P., Leonídio, A. R. A., Nascimento, G. M., \& Andrade, M. A. (2014). Microbiota intestinal das aves de produção. Enciclopédic Biosfera, Centro Científico Conhecer, Goiânia, 10(18), 2181. Recuperado de https://www.conhecer.org.br/enciclop/2014a/AGRARIAS/microbiota.pdf.

Garcia, G. D., Carvalho, M. A. R., Diniz, C. G., Marques, J. L., Nicoli, J. R., \& Farias, L. M. (2012). Isolation, identification and antimicrobial susceptibility of Bacteroides fragilis group strains recovered from broiler faeces. British Poultry Science, 53, 71-76. https://doi.org/0.1016/j.vetmic.2014.04.019.

Golder, H. M., Geier, M. S., Forder, R. E. A., Hynd, P. I., \& Hughes, R. J. (2011). Effects of necrotic enteritis challenge on intestinal micro-architecture and mucin profile. British Poultry Science, 52, 500-506. http://dx.doi.org/10,1080/00071668.2011.587183

Goldstein, E. J. C., Tyrrell, K. L., \& Citron, D. M. (2015). Lactobacillus species: taxonomic complexity and controversial susceptibilities. Clinical Infectious Diseases, 60(2), 98-107. https://doi.org/10.1093/cid/civ072

Guamán, R. A., Morocho, M. C., Yunga, V. H., Herrera, R. H., \& Sanchez, G. E. (2017). Cambios en la microbiota intestinal de las aves y sus implicaciones prácticas. Centro de Biotecnología, 6, 98-108. Recuperado de https://www.researchgate.net/publication/323152630_Cambios_en_la_microbiota_intestinal_de_las_aves_y_sus_implicaciones_practicas.

Gupta, R. S., Sawnani, S., Adeolu, M., Alnajar, S., \& Oren, A. (2018). Phylogenetic framework for the phylum Tenericutes based on genome sequence data: proposal for the creation of a new order Mycoplasmoidales ord. nov., containing two new families Mycoplasmoidaceae fam. nov. and Metamycoplasmataceae fam. nov. harbouring Eperythrozoon, Ureaplasma and five novel genera. Antonie Van Leeuwenhoek, 111(9), 1583-1630. https://doi.org/ 10.1007/s10482-018$1047-3$

Hazrati, S., Rezaeipour, V., \& Asadzadeh, S. (2020). Effects of phytogenic feed additives, probiotic and mannan-oligosaccharides on performance, blood metabolites, meat quality, intestinal morphology, and microbial population of Japanese quail. Brazilian Journal of Poultry Science, 61, 132-139. https://doi.org/ 10.1080/00071668.2019.1686122

Helmy, Y. A., Krücken, J., Abdelwhab, E. S. M., Himmelstjerna, G. S., \& Hafez, M. (2017). Molecular Diagnosis and Characterization of Cryptosporidium Spp. In Turkeys and Chickens in Germany Reveals Evidence for Previously Undetected Parasite Species. PLoS One, 12, e0177150. https://doi.org/10.1371/journal.pone.0177150

Holubova, N., Sak, B., Hlásková, L., Květoňova, D., Hanzal, V., Rajský, D., Rost, M., McEvoy, J., \& Kváč, M. (2018). Host Specificity and Age-Dependent Resistance to Cryptosporidium Avium Infection in Chickens, Ducks and Pheasants. Experimental Parasitology, 191, 62-65. https://doi.org/10.1016/j.exppara.2018.06.007

Hu, W., Zhang, W., Shah, S. W. A., Ishfaq, M., \& Li, J. 2021. Mycoplasma gallisepticum infection triggered histopathological changes, oxidative stress and apoptosis in chicken thymus and spleen. Developmental \& Comparative Immunology, 114, 103832. https://doi.org/10.1016/j.dci.2020.103832

Ishfaq, M., Zhang, W., Shah, S. W. A., Wu, Z., Wang, J., Ding, L., \& Li, J. (2019). The effect of Mycoplasma gallisepticum infection on energy metabolism in chicken lungs: Through oxidative stress and inflammation. Microbial Pathogenesis, 138, 103848. https://doi.org/10.1016/j.micpath.2019.103848

Kogut, M. H. (2018). O efeito da modulação do microbioma na saúde intestinal de aves domésticas. Ciência e Tecnologia de Ração Animal, $250,32-40$. https://doi.org/10.1016/j.anifeedsci.2018.10.008 
Kogut, M. H., \& Arsenault, N. J. (2016). Editorial: Gut Health: The New Paradigm in Food Animal Production. Frontiers in Veterinary Science, 31. https://doi.org/10.3389/fvets.2016.00071

Kollarcikova, M., Kubasova, T., Karasova, D., Crhanova, M., Cejkova, D., Sisak, F., \& Rychlik, I. (2019). Use of 16S rRNA gene sequencing for prediction of new opportunistic pathogens in chicken ileal and cecal microbiota. Poultry Science, 98, 2347-2353. https://doi.org/10.3382/ps/pey594

Kosmann, R. C. (2018). Impacto da adição dietética de antibiótico melhorador de desempenho e probiótico sobre a saúde intestinal e diversidade da microbiota intestinal de frangos de corte (Dissertação de Mestrado). Universidade Federal do Paraná, Palotina, Brasil.

Kraimi, N., Calandreau, L., Biesse, M., Rabot, S., Guitton, E., Velge, P., \& Leterrier, C. (2018). Absence of Gut Microbiota Reduces Emotional Reactivity in Japanese Quails (Coturnix japonica). Frontiers in Physiology, 9, 603. https://doi.org/10.3389/fphys.2018.00603

Lacey, J. A., Johanesen, P. A., Lyras, M., \& Moore, R. J. (2016). Diversidade genômica de cepas associadas à enterite necrótica de clostridium perfrigens: uma revisão. Patologia aviária, 45. https://doi.org/10.1080/03079457.2016.1153799

Lugli, G. A., Mangifesta, M., Duranti, S., Anzalone, R., Milani, C., Mancabelli, L., Alessandri, G., Turroni, F., Ossiprandi, MC., Sinderen, V., \& Ventura, M. (2018). Phylogenetic classification of six novel species belonging to the genus Bifidobacterium comprising Bifidobacterium anseris sp. nov., Bifidobacterium criceti sp. nov., Bifidobacterium imperatoris sp. nov., Bifidobacterium italicum sp. nov., Bifidobacterium margollesii sp. nov. and Bifidobacterium parmae sp. nov. Systematic and Applied Microbiology, 41, 173-183. https://doi.org/10.1016 / j.syapm.2018.01.002

Machado, L. S., Nascimento, E. R., Pereira, V. L. A., Abreu, D. L. C., \& Barreto, M. L. (2012). Revisão: Micoplasmoses aviárias. Enciclopédia Biosfera, Centro Científico Conhecer, Goiânia, 8(15). Recuperado de http://www.conhecer.org.br/enciclop/2012b/ciencias\%20agrarias/revisao.pdf

Mahrose, K. M., Elhack, M. E. A., Mahgoub, S. A., \& Attia, F. A. M. (2019). Influences of stocking density and dietary probiotic supplementation on growing Japanese quail performance. Anais da Academia Brasileira de Ciências, 91, e20180616. http://dx.doi.org/10.1590/0001-3765201920180616

Martínez, I., Perdicaro, D. J., Brown, A. W., Hammons, S., Carden, T. J., Carr, T. P., \& Walter, J. (2013). Alterações induzidas pela dieta do metabolismo do colesterol no hospedeiro provavelmente afetarão a composição da microbiota intestinal em hamsters. Microbiologia Aplicada e Ambiental, 79 , 516-524. https://doi.org/10.1128 / aem.03046-12

Oden, L. A., Lee, J. T., Pohl, S. K., Klein, A. E., Anderson, A. S., Dougherty, S. D., Broussard, C. T., Fitz-Coy, S. H., Newman, L. J., \& Caldwell, D. J. (2012). Influence of diet on oocyst output and intestinal lesion development in replacement broiler breeders following live oocyst coccidiosis vaccination. Journal of Applied Poultry Research, 21, 445-459. https://doi.org/10.3382/japr.2010-00264

Okamoto, A. S., Andreatti Filho, R. L., Rocha, T. S., \& Milbradt, E. L. (2011). Transference in vitro of the resistance to the antimicrobials between Escherichia coli, Lactobacillus spp. and Salmonella enteritidis isolated from chickens. Arquivo Brasileiro de Medicina Veterinária e Zootecnia, 63, 11491153. https://doi.org/10.1590/S0102-09352011000500015

Oliveira, A. V. B., Silva, R. A., Araújo, A. S., Brandão, P. A., \& Costa, F. B. (2011). Padrões microbiológicos da carne de frango de corte - referencial teórico. Revista Verde, 6(3), 1-16. Recuperado de https://www.gvaa.com.br/revista/index.php/RVADS/article/view/705/621

Paixão, L. A., \& Castro, F. F. S. (2016). A colonização da microbiota intestinal e sua influência na saúde do hospedeiro. Revista Ciência da Saúde, 14, 85-96. https://doi.org/10.5102/ucs.v14i1.3629

Pickler, L., Santin, E., \& Silva, A. V. S. (2011). Alternativas aos antibióticos para equilibrar a microbiota gastrointestinal de frangos. Archives of Veterinary Science, 16, 1-13. http://dx.doi.org/10.5380/avs.v16i3.18300

Quinn, P. J., Markey, B. K., Carter, M. E., Donnelly, W. J., \& Leonard, F. C. (2007). Microbiologia Veterinária e doenças infecciosas. (1a ed.). Jones \& Bartlett. Tradução Weiss, LHN e Weiss, DN. Artmed, Porto Alegre.

Ramakrishna, C., Kujawski, M., Chu, H., Li, L., Mazmanian, S. K., \& Cantin, E. M. (2019). Bacteroides fragilis polysaccharide A induces IL-10 secreting B and T cells that prevent viral encephalitis. Nature Communications, 10(1), 2153. https://doi.org/10.1038 / s41467-019-09884-6

Reis, T. L., \& Vieites, F. M. (2019). Antibiótico, prebiótico, probiótico e simbiótico em rações de frangos de corte e galinhas poedeiras. Ciência Animal, 29(3), 133-147. Recuperado de http://www.uece.br/cienciaanimal/dmdocuments/x\%2005.\%20REVIS\%C3\%83O\%20DE\%20LITERATURA\%202019.pdf

Sahin, O., Kassem, I. I., Shen, Z., Lin, J., Rajashekara, G., \& Zhang, Q. (2015). Campylobacter in Poultry: Ecology and Potential Interventions. Avian Diseases, 59, 185-200. https://doi.org/10.1637/11072-032315-Review

Salwan, R. \& Sharma, V. (2020). Molecular and biotechnological aspects of secondary metabolites in actinobacteria. Microbiological Research, 231 , e126374. https://doi.org/10.1016/j.micres.2019.126374

Schets, F. M., Jacobs-Reitsma, W. F., Plaats, R. Q. J., Heer, L. K., Hoek, A. H. A. M., Hamidjaja, R. A., Husman, A. M. R., \& Blaak, H. (2017). Prevalence and Types of Campylobacter on Poultry Farms and in Their Direct Environment. Journal Water Health, 15, 849-862. https://doi.org/10.2166/wh.2017.119

Shojadoost, B., Vince, A. R., \& Prescott, J. F. (2012). A indução experimental bem-sucedida de enterite necrótica em frangos por Clostridium perfringens: uma revisão crítica. Pesquisa Veterinária, 43, 74. https://doi.org/10.1186 / 1297-9716-43-74

Shokryazdan, P., Jahromi, M. F. J., Liang, J. B., Ramasamy, K., Sieo, C. C., \& Ho, Y. W. (2017). Effects of a Lactobacillus salivarius mixture on performance, intestinal health and serum lipids of broiler chickens. PLoS One, 12(5): e0175959. https://doi.org/10.1371/journal.pone.0175959

Skennerton, C. T., Haroon, M. F., Briegel, A., Shi, J., Jensen, G. J., Tyson, G. W., \& Orphan, V. J. (2016). Phylogenomic analysis of Candidatus 'Izimaplasma' species: free-living representatives from a Tenericutes clade found in methane seeps. The ISME Journal, 10(11), 2679-2692. https://doi.org/10.1038/ismej.2016.55

Sommer, F., \& Bäckhed, F. (2013). The gut microbiota - masters of host development and physiology. Nature Reviews Microbiology, 11, 227-238. https://doi.org/10.1038/ nrmicro2974 
Research, Society and Development, v. 11, n. 2, e22411225583, 2022

(CC BY 4.0) | ISSN 2525-3409 | DOI: http://dx.doi.org/10.33448/rsd-v11i2.25583

Souza, L. T. (2017). Clostridium perfringens: Uma revisão (Dissertação de Mestrado). Universidade Federal de Minas Gerais, Belo Horizonte, Brasil.

Su, H., McKelvey, J., Rollins, D., Zhang, M., Brightsmith, D. J., Derr, J., \& Zhang, S. (2014). Cultivable Bacterial Microbiota of Northern Bobwhite (Colinus virginianus): A New Reservoir of Antimicrobial Resistance?. Plos One, 9, e99826. https://doi.org/10.1371 / journal.pone.0099826

Sureshkumar, S., Lee, H. C., Jung, S. K., Kim, D., Oh, K. B., Yang, H., Jo, Y. J., Lee, H. S., Lee, S., \& Byun, S. J. (2020). Inclusion of Lactobacillus salivarius strain revealed a positive effect on improving growth performance, fecal microbiota and immunological responses in chicken. Archivos Microbiology, 203(2), 847-853. https://doi.org/10.1007/s00203-020-02088-3

Wang, Y., Huang, Jiao-Mei., Zhou, Ying-Li., Almeida, A., Finn, R. D., Danchin, A., \& He, Li-Sheng. (2020). Phylogenomics of expanding uncultured environmental Tenericutes provides insights into their pathogenicity and evolutionary relationship with Bacilli. BMC Genomics, 21(1), 408. https://doi.org/10.1186/s12864-020-06807-4

Wilkinson, N., Hughes, R. J., Aspden, W. J., Chapman, J., Moore, R. J., \& Stanley, D. (2016). The gastrointestinal tract microbiota of the Japanese quail, Coturnix japonica. Applied Microbiology Biotechnology, 100, 4201-4209. https://doi.org/10.1007/s00253-015-7280-z

Xiao, Y., Xiang, Y., Zhou, W., Chen, J., Li, K., \& Yang, H. (2017). Microbial community mapping in intestinal tract of broiler chicken. Poultry Science, 96, 1387-1393. http://dx.doi.org/10.3382 / ps / pew372

Xu, B., Liu, R., Ding, M., Zhang, J., Sun, H., Liu, C., Lu, F., Zhao, S., Pan, Q., \& Zhang, X. (2020). Interaction of Mycoplasma synoviae with chicken synovial sheath cells contributes to macrophage recruitment and inflammation. Poultry Science, 99(11), 5366-5377. https://doi.org/10.1016/j.psj.2020.08.003

Zawadzki, F. (2016). Microbiologia Veterinária Aplicada. (3a ed.) Editora e Distribuidora Educacional S.A. Guanabara Koogan, Londrina.

Zebeli, B. U. M., Magowan, E., Hollmann, M., Ball, M. E. E., Molnár, A., Witter, K., Ertl, R., Hawken, R. J., Lawlor, P. G., O'Connell, N. E., Aschenbach, J., \& Zebeli, Q. (2018). Differences in intestinal size, structure, and function contributing to feed efficiency in broiler chickens reared at geographically distant locations. Poultry Science, 97, 578-591. https://doi.org/10.3382 / ps / pex332

Zhang, W., Liu, Y., Zhang, Q., Shah, S. W. A., Wu, Z., Wang, J., Ishfaq, M., \& Li, J. (2020). Mycoplasma gallisepticum Infection Impaired the Structural Integrity and Immune Function of Bursa of Fabricius in Chicken: Implication of Oxidative Stress and Apoptosis. Frontiers Veterinary Science, 7, 225. https://doi.org/10.3389/fvets.2020.00225

Zhou, Bian-Hua., Jia, Liu-Shu., Wei, Shan-Shan., Ding, Hai-Yan., Yang, Jing-Yun., \& Wang, Hong-Wei. (2020). Effects of Eimeria tenella infection on the barrier damage and microbiota diversity of chicken cécum. Poultry Science, 99(3), 1297-1305. https://doi.org/10.1016/j.psj.2019.10.073

Zhou, H., Yu, B., Gao, J., Htoo, J. K., \& Chen, D. (2018). Regulation of intestinal health by branched-chain amino acids, Animal Science Journal, 89, 3-11. https://doi.org/10.1111/ asj.12937 